\title{
Influence of Self-Esteem and Psychiatric Diagnosis on Health-Related Quality of Life in Children and Adolescents with School Refusal Behavior
}

This article was published in the following Dove Press journal:

Neuropsychiatric Disease and Treatment

\author{
Hiroki Matsuura $\mathbb{D}^{\prime}$ \\ Hidemi Iwasaka ${ }^{2}$ \\ Satoko Nezu ${(\mathbb{D})^{3}}^{4}$ \\ Toyosaku Ota (iD ${ }^{4}$ \\ Kosuke Okazaki ${ }^{4}$ \\ Kazuhiko Yamamuro ${ }^{4}$ \\ Yoko Nakanishi ${ }^{5}$ \\ Naoko Kishimoto ${ }^{4}$ \\ Junzo lida ${ }^{6}$ \\ Toshifumi Kishimoto ${ }^{4}$ \\ 'Department of Psychiatry, Nara \\ Prefecture General Rehabilitation Center, \\ Shiki-Gun, Nara, Japan; ${ }^{2}$ Developmental \\ Center for Child and Adult, Shigisan \\ Hospital, Ikoma-Gun, Nara, Japan; \\ ${ }^{3}$ Department of Epidemiology, Nara \\ Medical University, Kashihara, Nara, \\ Japan; ${ }^{4}$ Department of Psychiatry, Nara \\ Medical University, Kashihara, Nara, \\ Japan; ${ }^{5}$ Department of Psychiatry, Akitsu \\ Kounoike Hospital, Gose, Nara, Japan; \\ ${ }^{6}$ Faculty of Nursing, Nara Medical \\ University, Kashihara, Nara, Japan
}

Correspondence: Toyosaku Ota Department of Psychiatry, Nara Medical University, 840 Shijyo-Cho, Kashihara,

Nara 634-8522, Japan

Tel +8I-744-22-305।

Fax +8I-744-22-3854

Email toyosaku@naramed-u.ac.jp
Background: School refusal behavior (SRB), which is the refusal to attend or remain in school, has been associated with emotional, psychological, and other behavioral problems, as well as a lower health-related quality of life (HRQOL). However, the effects of self-esteem and a psychiatric diagnosis in students with SRB on HRQOL are not yet known. Understanding these relationships could help to develop more effective therapeutic interventions.

Methods: A total of 175 young people (aged 8-18 years old) who visited our medical centers and outpatient clinics participated in the study. This comprised the SRB group $(n=70)$ and an age- and sex-matched control group $(\mathrm{n}=105)$. Information about any psychiatric diagnosis was collected from medical records, HRQOL was measured using the J-KIDSCREEN-52, SRB was assessed using the School Refusal Assessment Scale-Revised for Japanese Attendance at School, self-esteem was measured using the Rosenberg Self-Esteem Scale, children's mental health status was measured using the Strengths and Difficulties Questionnaire, and social support was measured using the Oslo 3-item Social Support Scale. We performed between-group comparisons and multiple regression analysis.

Results: The SRB had a significantly lower HRQOL than the control group in several of the KIDSCREEN-52 dimensions. In the SRB group, 35.7\% had chronic disease and $35.7 \%$ of their parents had health problems and were receiving treatment. The multiple regression analysis revealed that, within the SRB group, a psychiatric diagnosis was associated with a lower HRQOL. However, this was not the case for a diagnosis of autistic spectrum disorder. Self-esteem positively affected HRQOL in six dimensions of the KIDSCREEN52 within the SRB group.

Conclusion: Our results could inform the development of support strategies for young people with SRB. Namely, support that enhances self-esteem could be used to increase HRQOL in young people with SRB. Furthermore, the presence of psychiatric disorders should be assessed as early as possible.

Keywords: school refusal behavior, health-related quality of life, KIDSCREEN, Rosenberg self-esteem scale, autism spectrum disorder

\section{Introduction}

School Refusal Behavior (SRB) is defined as child-motivated refusal to attend school and/or difficulties remaining in class for an entire day. ${ }^{1,2}$ This definition covers behavior associated with (1) students who want to go to school but stay home out of fear or anxiety, often described as school refusal, and (2) students who skip school because of defiant behavior or lack of interest in school, also referred to as truancy. ${ }^{2}$ The prevalence of anxious school refusal is reported to be $2 \%$ in the 
U.S. ${ }^{3}$ However, estimating the prevalence of SRB is difficult because SRB includes partial or delayed absences. The prevalence of SRB is estimated at around $8.2 \%$ in a community sample including anxiety-based school refusal and truancy. ${ }^{3-5}$ Although SRB is a relatively recent topic, to our knowledge, there have been no published reports that describe the health-related quality of life (HRQOL) of children with SRB who have visited medical services.

The Japanese Ministry of Education, Culture, Sports, Science and Technology (MEXT) defined not-attendance at school as [when] students do not attend or are unable to attend school because of psychological, emotional and physical reasons, or sociological factors and background, but not disease or economic reasons.

The MEXT definition of not-attendance at school and the definition of SRB both include truancy. The MEXT have investigated students who were absent from school for more than 30 days within a given year since 1991, in research on issues about guidance for teachers on student misbehavior. They reported that 164,528 students, which represents $1.7 \%$ of elementary and secondary school students, were absent from school for more than 30 days in 2018. The proportion of elementary and secondary school students exhibiting non-attendance at school has been increasing since $1992,{ }^{6}$ and the issue of not-attendance at school is serious for the Japanese education system.

Characteristics of individuals with SRB have been reported to be poor relationships with peers and difficulties in making friends because of shyness, aggression, or withdrawal. $^{3,7}$ Ohmann et al reported that children with prolonged SRB treated with a cognitive behavioral group therapy program displayed impaired executive functions. ${ }^{8}$ In the general child and adolescent population, studies of SRB have reported high rates of emotional and behavioral problems. ${ }^{2,3}$ Honjo et al reported that a large proportion of school refusers expressed somatic complaints together with depression. ${ }^{9}$ Prabhuswamy et al reported that of 33 students with school refusal aged 8 to 16 years, 29 subjects $(87.9 \%)$ had a psychiatric diagnosis. Depressive disorder (63.6\%) was the most common, followed by specific phobias $(30.3 \%) .{ }^{10}$ In a clinical sample $(n=143)$ of youths $(5-17$ years $)$ with $\mathrm{SRB}$, the most common primary diagnoses included separation anxiety disorder $(22.4 \%)$, generalized anxiety disorder (10.5\%), oppositional defiant disorder (8.4\%), and depression $(4.9 \%){ }^{2}$ Kurita et al reported that SRB is significantly more common in students with Autism Spectrum Disorder (ASD) compared with typically developing students. ${ }^{11,12}$
Based on our clinical experience, we believe that increasing self-esteem or self-efficacy is needed to reintegrate children with SRB into society. Otto et al reported that self-efficacy is positively associated with HRQOL assessed by the KIDSCREEN-10. ${ }^{13}$ Haraldstad et al reported that being involved in bullying, as either a victim or a bully, is associated with a lower HRQOL. Increasing self-efficacy may be one way to increase HRQOL in adolescents. ${ }^{14}$ Heyne et al reported that cognitive-behavioral therapy for children with school refusal decreases the fears surrounding attending school. Cognitive-behavioral therapy has been found to boost the mediating effect of self-efficacy on school refusal. ${ }^{15}$ Previous reports have shown that the number of days of absence is associated with a lower HRQOL in the general population. ${ }^{16}$ In recent study, Gonzálvez et al reported that social functioning acted as a protective factor of SRB due to anxiety symptoms or feelings of negative affect linked to the obligation to attend school. ${ }^{17}$

We made three hypotheses: 1) The HRQOL of students with SRB would be lower than that of the control group; 2) Students with SRB who are diagnosed with a psychiatric disorder would have a lower HRQOL than students with SRB who have no diagnosis; and 3) High self-esteem increases HRQOL of students with SRB. HRQOL, psychiatric diagnoses and self-esteem of students with SRB were measured, and how psychiatric diagnoses and self-esteem affect their HRQOL was investigated.

\section{Methods}

\section{Participants}

\section{Control Group}

Self-reported and parent-reported versions of J-KIDSCREEN-52 and The Oslo 3-item Social Support Scale (OSS-3) were measured to assess the HRQOL and social support of 325 children without chronic diseases who visited one general hospital and six outpatient clinics because of infections or vaccination. The inclusion criterion for the control group was an age between 8 and 18 years. The exclusion criteria were children and adolescents with a chronic disease or SRB. Of 325 children without chronic disease or SRB, 105 were recruited as the age- and sex-matched control group.

\section{SRB Group}

We recruited 123 children with SRB (8-18 years old) who first visited the Nara Medical University outpatient clinic or Nara Prefectural General Medical Center because of 
SRB between April 2015 and March 2018. A total of 70 out of 123 children responded (a response rate of 56.9\%). We distributed a questionnaire that measured HRQOL (self-reported and parent-reported version), social support, adaptation, and self-esteem to be completed at home by children with SRB and their parents, and collected them after 4 weeks. The inclusion criteria were an age of 8-18 years and the presence of SRB detected when visiting our medical service. The exclusion criterion was children who had started pharmacotherapy for SRB or another psychiatric condition before assessment. Participation was voluntary, and a written informed consent letter was obtained from all participants and their parents. If consent was not obtained, we did not to notify the treating doctor.

\section{Measures}

\section{Psychiatric Diagnoses}

Experienced child and adolescent psychiatrists diagnosed psychiatric disorders according to the ICD-10. ${ }^{18}$ Information about treatment was obtained by medical records and a written report of the doctors in charge of their treatment.

\section{J-KIDSCREEN-52}

The KIDSCREEN-52 was developed from the KIDSCREEN project funded by the European Commission (2001-2004), and measures children's perspective on their own health status. ${ }^{19}$ The KIDSCREEN-52 comprises 52 items, each of which is categorized into one of the following ten dimensions: Physical Well-being (PH), Psychological Well-being (PW), Moods and Emotions (ME), Self-Perception (SP), Autonomy (AU), Parent Relationships and Home Life (PA), Financial Resources (FI), Social Support and Peers (PE), School Environment (SC), and Social Acceptance (SA). Participants are asked to respond to items according to their feelings and experiences in the last week. The KIDSCREEN-52 assesses either the frequency of a behavior/feeling or the intensity of an attitude using a 5-point Likert response scale. Negatively formulated items are reversed and scores are summed for each of the 10 dimensions. The scores for each dimension are transformed into T-scores with a mean of 50 and a standard deviation (SD) of 10 , based on a representative sample of the European general population. ${ }^{20}$ The KIDSCREEN-52 is advantageous in that it (1) is applicable to a cross-cultural setting because it was developed simultaneously in several different countries and was subsequently tested with a large number of representatives of people across different countries and cultures, (2) covers the physical, psychological, social, and behavioral aspects of well-being and functioning from a child's point of view, (3) has corresponding versions (self-reported and parent-reported versions) in which each item of the parent version is reworded to the self (child)reported questionnaire. We used self-reported and parentreported versions. The questionnaire can be completed in approximately 10-20 mins, and can be completed by young people between 8 and 18 years old. Acceptable levels of reliability and validity were confirmed by Nezu et al. ${ }^{21}$

\section{The School Refusal Assessment Scale-Revised for Japanese Attendance at School}

The School Refusal Assessment Scale-Revised for Japanese Attendance at School (SRAS-R-JA) is a 20-item child version that was revised so that students without SRB can answer the questionnaire. Items are scored on a 5-point (1-5) Likert-type scale. Tsuchiya et al confirmed the reliability and validity of the SRAS-R-JA in 6th-grade elementary school children. The SRAS-R-JA total score is correlated with the number of days of absence ${ }^{22}$ and is indicative of persistent SRB. The SRAS-R-JA assesses the presence and severity of four aspects of SRB, as follows: avoidance of school-related stimuli that provoke negative emotion; escape from aversive social and/or situations; pursuit of attention from significant others; and pursuit of tangible reinforcers outside of school. ${ }^{23-25}$

\section{The Rosenberg Self-Esteem Scale}

The Rosenberg self-esteem scale (RSES) is a 10-item measure that was developed by Rosenberg. ${ }^{26}$ We used the Japanese version translated by Yamamoto et $\mathrm{al}^{27}$ The RSES assesses self-esteem, and participants rate items such as "I feel good enough" using a 5-point Likert response scale. The total score is $10-50$ points, and a higher score indicates a higher selfesteem. The validity of the Japanese version of the RSES has been confirmed by Sakurai. ${ }^{28}$

\section{The Strengths and Difficulties Questionnaire}

The Strengths and Difficulties Questionnaire (SDQ) is a brief behavioral screening questionnaire for young people aged 3-16 years old that was developed by Goodman to assess children's mental health status. ${ }^{29,30}$ The SDQ items are scored on a 3-point Likert response scale. There are five items for each of the five dimensions, which include emotional symptoms, conduct problems (behavioral problems), hyperactivity/inattention, peer relationship problems, and prosocial behavior. The Japanese version of the SDQ includes 25 items and can be downloaded from the website http://www.sdqinfo.org/. Parents completed the SDQ 
questionnaires. The Total Difficulty Score (TDS) is the sum of scores for all dimensions except prosocial behavior. A high score in the prosocial behavior domain indicates a better mental health status, and a high score in the other four dimensions is indicative of worse mental health status. We used the cutoff points reported by Noda et $\mathrm{al}^{31}$ as follows: $>$ three points indicate abnormal in emotional symptoms and conduct problems, $>$ five points indicate abnormal in hyperactivity/inattention, $>$ two points indicate abnormal in peer relationship problems, $<$ four points indicate abnormal in prosocial behavior. The SDQ classifies respondents as normal, borderline, abnormal.

\section{The Oslo 3-Item Social Support Scale}

The Oslo 3-item Social Support Scale was adapted to assess the level of social support. This scale measures (1) how many people provide a sense of security and support to the child (1 item) and (2) the level of emotional and instrumental support provided by those people (2 items). The sum of these three items is the total score, which ranges from 3 to 14 points, whereby a higher score indicates stronger social support. The questionnaire was translated into Japanese by Nezu et al. ${ }^{21}$

\section{Ethics Approval and Consent to Participate}

The present study protocol was approved by the Ethics Committee of Nara Medical University and Nara Prefecture General Medical Center. Written informed consent letters were obtained from each participating student who was at least 18 years of age. If the student was under 18 years of age, the purpose and nature of the study were explained to the student and his/her parents, and a written informed consent letter was obtained from one of the student's parents. This study was conducted in accordance with the Declaration of Helsinki.

\section{Statistical Analyses}

All statistical analyses were performed using SPSS 24.0 (IBM Corporation, IBM SPSS Statistics, Chicago, Illinois, USA). Differences in mean T-scores between the SRB group and the control group were tested using the Student's $t$-test within each of the J-KIDSCREEN-52 dimensions, $t$-tests were also used to compare age and number of family members and siblings between the SRB group and the control group. The Chisquare test was used to explore between-group differences in background characteristics. Differences in mean T-scores between the self-report and proxy-reported J-KIDSCREEN52 were evaluated using the Student's $t$-test for each dimension. Internal consistency of each dimension of J-KIDSCREEN-52, RSES, SRAS-R-JA, SDQ, OSS-3 were evaluated by Cronbach's alpha coefficients. We examined the Intraclass Correlation Coefficient (ICC) between scores of the self-reported and proxy-reported questionnaire using a oneway random effects model to determine test-retest reliability. A coefficient of $\geqq 0.60$ was considered as evidence for adequate test-retest stability. ${ }^{32}$ A $P$-level of $<0.05$ was considered as statistically significant. Multiple regression analyses were performed with J-KIDSCREEN-52 dimension scores as dependent variables, and with the RSES, SRAS-R-JA total score, and psychiatric diagnoses as independent variables. We used dummy variables for psychiatric diagnoses, including F3 (except for F3 = 0, F3 = 1), F4 (except for F4 = 0, F4 = 1), F8 (except for $\mathrm{F} 8=0, \mathrm{~F} 8=1$ ), and no diagnosis (any diagnosis $=0$, no diagnosis $=1)$. The potential bias due to multicollinearity seemed negligible $(1.000<$ variance inflation factor $<1.174)$. We used a stepwise method for the selection of variables, and adjusted $\mathrm{R}^{2}$ was used for judgement of goodness of fit.

\section{Results}

\section{Demographic and Clinical Characteristics of Children and Parents in the SRB Group}

The mean age of the SRB group was $13.5 \pm 2.4$ years (range, 8-18 years), and 50 patients were $13-18$ years of age (71.4\%). Thirty-two patients had weak social support as assessed by the OSS-3 in the SRB group. Internal consistency of OSS-3 was 0.61 . Twenty-five patients $(35.7 \%)$ in the SRB group had chronic disease, and no children in the control group had a chronic disease (chi-square, $P<0.001$ ). Twenty-five parents (35.7\%) had an illness for which they were currently receiving treatment, and among these, 7 parents $(10 \%)$ had a mental disorder in the SRB group. Eleven parents $(10.5 \%)$ had illness for which they were currently receiving treatment in the control group. In the SRB group prevalence of illness of parents were higher than that of control groups (chi-square, $P<0.001$ ). A total of 45 students $(64.3 \%)$ were absent from school for over 30 days per year in the SRB group, and no student was absent from school for over 30 days per year in the control group (chisquare, $P<0.001)$. Twelve students $(17.1 \%)$ were from single-parent families in the SRB group (Table 1).

In the SRB group, a total of $33.3 \%$ were exhibited notattendance at school and a total of $29.2 \%$ complained of physical symptoms as a chief symptom of SRB at their first visit to medical services. A total of $43.1 \%$ reported difficulties with friend relationships, and $16.7 \%$ reported 
Table I Characteristics and Social Support of the SRB and Control Groups

\begin{tabular}{|c|c|c|c|c|c|c|}
\hline & & & $\begin{array}{l}\text { SRB } \\
(N=70)\end{array}$ & $\begin{array}{l}\text { Control } \\
(\mathrm{N}=105)\end{array}$ & $\begin{array}{l}\text { Chi-Square } \\
\text {-Value }\end{array}$ & $\begin{array}{l}\text { t-test } \\
\text { P-value }\end{array}$ \\
\hline Self Answering & $\begin{array}{l}\text { Age } \\
\text { Sex } \\
\text { Chronic-diseased } \\
\text { OSS-3 }\end{array}$ & $\begin{array}{l}\text { Mean (SD) } \\
8-12 \text { y.o., N (\%) } \\
\text { I3-18 y.o., N (\%) } \\
\text { Female, N (\%) } \\
\mathrm{N}(\%) \\
\text { Weak N (\%) }\end{array}$ & $\begin{array}{l}13.5(2.4) \\
20(28.6) \\
50(71.4) \\
27(38.6) \\
25(35.7) \\
32(45.7)\end{array}$ & $\begin{array}{l}13.4(2.5) \\
30(28.6) \\
75(71.4) \\
42(40) \\
0(0) \\
36(34.3)\end{array}$ & $\begin{array}{l}I \\
0.666 \\
<0.00 I * \\
0.083\end{array}$ & 0.88 \\
\hline $\begin{array}{l}\text { Proxy answering (parent } \\
\text { answering) }\end{array}$ & $\begin{array}{l}\text { Number of siblings } \\
\text { Number of family members } \\
\text { (excluding the person) } \\
\text { Low educational level of mothers } \\
\text { Parental feeling of poverty } \\
\text { Parental health problems under } \\
\text { treatment } \\
\text { Single-parent } \\
\text { Days of absence per year }\end{array}$ & $\begin{array}{l}N(S D) \\
N(S D) \\
\text { Less than } 12 \\
\text { years } N(\%) \\
\text { Poor N (\%) } \\
N(\%) \\
N(\%) \\
\text { More than } \\
\text { 30days } N(\%)\end{array}$ & $\begin{array}{l}2.2(0.8) \\
3.2(1.1) \\
4(5.7) \\
14(20.0) \\
25(35.7) \\
12(17.1) \\
45(64.3)\end{array}$ & $\begin{array}{l}2.2(0.8) \\
3.7(1.1) \\
5(4.8) \\
14(13.3) \\
11(10.5) \\
8(7.6) \\
0(0)\end{array}$ & $\begin{array}{l}0.756 \\
0.182 \\
<0.00 I^{*} \\
0.054 \\
<0.001^{*}\end{array}$ & $\begin{array}{l}0.356 \\
0.138\end{array}$ \\
\hline
\end{tabular}

Note: $*_{p}<0.05$.

Abbreviation: OSS-3, The Oslo 3-item Social Support Scale.

that their illness was the trigger of SRB. A total of $44.4 \%$ had been absent from school before visiting the medical services. A total of $20.8 \%$ had poor school records. The mean duration between the day that they exhibited SRB and the day of the first medical visit was $177.8 \pm 201$ days.

Concerning the psychiatric diagnoses, 36 patients had neurosis (51.4\%; F4 disorders), 4 had depressive episodes (5.7\%; F3 disorder), 10 patients (14.3\%) had F8 disorders (ASD and Learning Disorder), 3 patients (4.3\%) had hyperkinetic disorder, 1 patient exhibited stuttering, and 8 patients $(11.4 \%$ ) had no diagnoses (Table 2 ).

\section{Primary Endpoint}

Each HRQOL dimension between the SRB group and the control group was compared. The SRB group had significantly lower scores in nine dimensions of the J-KIDSCREEN-52 compared with control group, including Physical Well-being $(P<0.001,95 \%$ CI -24.5 to -17.7$)$, Psychological Well-being $(P<0.001,95 \%$ CI -21.2 to -14.0), Moods and Emotions $(P<0.001,95 \% \mathrm{CI}-16.2$ to -10.2), Self-Perception ( $P<0.001,95 \% \mathrm{CI}-6.9$ to -2.0$)$, Autonomy $(P<0.001$, 95\% CI,-12.1 to -6.8$)$, Parent Relationships and Home Life $(P<0.001,95 \% \mathrm{CI}-11.5$ to -5.1), Financial Resources $(P=0.014,95 \% \mathrm{CI}-8.4$ to -1.0), Social Support and Peers $(P<0.001,95 \%$ CI -32.8 to 23.8), and School Environment $(P<0.001,95 \% \mathrm{CI}-23.8$ to -15.1) Cronbach's alpha values for each of the ten dimensions were $0.30(\mathrm{PH}), 0.92$ (PW), 0.82 (ME), 0.45 (SE), 0.65 (AU), 0.86 (PA), 0.89 (FI), 0.91 (PE), 0.49 (SC), and 0.65 (AS) in SRB group (Table 3, Figure 1).

In the SRB group, self-reported HRQOL was higher than the proxy-reported HRQOL in the dimensions of Physical Well-being $(\Delta=4.49)$, Moods and Emotions $(\Delta=6.17)$, Self-Perception $(\Delta=2.43)$, Parent Relationships and Home Life $(\Delta=5.02)$, and School Environment $(\Delta=5.58)$. The ICC between selfreported and proxy-reported HRQOL was slight to fair (range, 0.094-0.386) in the dimensions of Psychological Well-being, Moods and Emotions, Autonomy, Parent Relationships and Home Life, Financial Resources, and Social Acceptance. There was a significant difference between self-reported HRQOL and proxy-reported HRQOL in the dimensions of Moods and Emotions and Parent Relationships and Home Life. We also found a low ICC between selfreported HRQOL and proxy-reported HRQOL in the dimensions of Moods and Emotions and Parent Relationships and Home Life. In the control group, the ICC between self-reported and proxy-reported HRQOL was fair (range, 0.365-0.389) in the dimensions of Self-Perception, Autonomy, and Parent Relationships and Home Life (Table 4). 
Table 2 Characteristics and Psychiatric Diagnoses Assessed by the ICD-IO in the SRB Group

\begin{tabular}{|c|c|c|c|}
\hline & & $\mathbf{N}$ & $\%$ \\
\hline Chief symptoms & $\begin{array}{l}\text { School refusal } \\
\text { Somatic symptoms } \\
\text { Depression } \\
\text { Sleep disorder } \\
\text { Anxiety } \\
\text { Developmental disorder } \\
\text { Anthropophobia } \\
\text { Others }\end{array}$ & $\begin{array}{l}24 \\
21 \\
5 \\
5 \\
5 \\
4 \\
2 \\
4\end{array}$ & $\begin{array}{l}33.3 \\
29.2 \\
6.9 \\
6.9 \\
6.9 \\
5.6 \\
2.8 \\
5.6\end{array}$ \\
\hline Trigger of SRB & $\begin{array}{l}\text { Relationships with friends } \\
\text { Illness } \\
\text { Relationship with teachers } \\
\text { Impaired lifestyle habit } \\
\text { Learning problems } \\
\text { Cannot make friends } \\
\text { School rules } \\
\text { Relationship with parents } \\
\text { Conflict in club activities } \\
\text { Family environment } \\
\text { Discord in house } \\
\text { Internet, gaming problems } \\
\text { Others }\end{array}$ & $\begin{array}{l}31 \\
12 \\
9 \\
7 \\
6 \\
6 \\
4 \\
4 \\
3 \\
2 \\
1 \\
1 \\
18\end{array}$ & $\begin{array}{l}43.1 \\
16.7 \\
12.5 \\
9.7 \\
8.3 \\
8.3 \\
5.6 \\
5.6 \\
4.2 \\
2.8 \\
1.4 \\
1.4 \\
25\end{array}$ \\
\hline $\begin{array}{l}\text { Past history of SRB } \\
\text { Frequency of attendance per } \\
\text { week }\end{array}$ & $\begin{array}{l}0 \text { day } \\
\text { 1-2 days } \\
3-4 \text { days } \\
5-6 \text { days }\end{array}$ & $\begin{array}{l}32 \\
46 \\
17 \\
5 \\
2\end{array}$ & $\begin{array}{l}44.4 \\
63.9 \\
23.6 \\
6.9 \\
2.8\end{array}$ \\
\hline School records & $\begin{array}{l}\text { Good } \\
\text { Moderate } \\
\text { Poor }\end{array}$ & $\begin{array}{l}7 \\
46 \\
15\end{array}$ & $\begin{array}{l}9.7 \\
63.9 \\
20.8\end{array}$ \\
\hline $\begin{array}{l}\text { Psychiatric diagnoses (ICD-I0) } \\
\text { F3 }\end{array}$ & Depression & 4 & 5.7 \\
\hline $\mathrm{F} 4$ & $\begin{array}{l}\text { Undifferentiated Somatoform } \\
\text { disorder } \\
\text { Adjustment disorder } \\
\text { Conversion } \\
\text { Obsessive-compulsive } \\
\text { disorder } \\
\text { Other specific anxiety disorder }\end{array}$ & $\begin{array}{l}17 \\
14 \\
3 \\
1 \\
1\end{array}$ & $\begin{array}{l}24.2 \\
20 \\
4.2 \\
1.4 \\
1.4\end{array}$ \\
\hline F8 & $\begin{array}{l}\text { Autism spectrum disorder } \\
\text { Learning disorder }\end{array}$ & $\begin{array}{l}9 \\
1\end{array}$ & $\begin{array}{l}12.9 \\
1.4\end{array}$ \\
\hline F9 & $\begin{array}{l}\text { Hyperkinetic disorders } \\
\text { Stutter }\end{array}$ & $\begin{array}{l}3 \\
1\end{array}$ & $\begin{array}{l}4.2 \\
1.4\end{array}$ \\
\hline Others & $\begin{array}{l}\text { Sleeping rhythm disorder } \\
\text { Schizophrenia spectrum } \\
\text { disorder } \\
\text { No diagnosis } \\
\text { Borderline intellectual disorder }\end{array}$ & $\begin{array}{l}3 \\
1 \\
8 \\
1\end{array}$ & $\begin{array}{l}4.2 \\
1.4 \\
11.4 \\
1.4\end{array}$ \\
\hline
\end{tabular}

Abbreviation: SRB, School Refusal Behavior.
In the SRB group, the TDS score indicated that 35 patients $(52.2 \%)$ had abnormal mental health status. Internal consistency of SDQ was 0.58 (Figure 2).

A high RSES score was associated with higher J-KIDSCREEN-52 scores in the dimensions of Physical Well-being, Moods and Emotions, Self-Perception, Parent Relationships and Home Life, and Financial Resources (standard partial regression coefficient: $\beta=0.338,0.338$, $0.663,0.405,0.361,0.298,0.447$, respectively). The association between RSES score and Moods and Emotions was particularly prominent. The SRAS-R-JA total score was associated with a lower J-KIDSCREEN-52 in the dimensions of Social Support and Peers, School Environment, and Social acceptance $(\beta=-0.378,-0.481,-0.557$, respectively). An F3 diagnosis (depression) was associated with a lower J-KIDSCREEN-52 in the dimensions of Physical Well-being, Autonomy, and Social Support and Peers $(\beta=-0.229,-0.255,-0.253$, respectively). An F4 diagnosis (undifferentiated somatoform disorders and adjustment disorder) was associated with a lower J-KIDSCREEN-52 in the dimension of Physical Wellbeing $(\beta=-0.389)$. An F8 diagnosis (ASD) was associated with a higher J-KIDSCREEN-52 in the dimensions of Psychological Well-being and Autonomy $(\beta=0.29$, 0.262). SRB with no diagnosis was not associated with any dimensions of the J-KIDSCREEN-52. The associations were weak $\left(\mathrm{R}^{2}=0.072\right.$ for Financial Resources to $\mathrm{R}^{2}=0.434$ for Mood and Emotions), but the association between the RSES and the J-KIDSCREEN-52 dimension of Moods and Emotions was prominent (Table 5).

\section{Discussion}

We found that HRQOL of children with SRB was lower than control group in many dimensions and that they need assistance highly. We also found that self-esteem could be a protective factor for HRQOL in children with SRB that visited medical services. We made three hypotheses: 1) The HRQOL of students with SRB would be lower than that of the control group; 2) Students with SRB who are diagnosed with a psychiatric disorder would have a lower HRQOL than students with SRB who have no diagnosis; and 3) High self-esteem increases HRQOL of students with SRB.

The first hypothesis was partially supported. In this study, because few children were bullied, HRQOL in the Social Acceptance dimension was maintained. In past 
Table 3 Between-Group Comparison of HRQOL Assessed by the J-KIDSCREEN-52

\begin{tabular}{|c|c|c|c|c|c|c|c|c|c|c|}
\hline & \multicolumn{4}{|l|}{ SRB $(N=70)$} & \multicolumn{3}{|c|}{ Control $(N=105)$} & & & \multirow{3}{*}{$P$-value } \\
\hline & \multirow[t]{2}{*}{$\begin{array}{l}\text { Number of } \\
\text { Items }\end{array}$} & & & $\begin{array}{l}\text { Internal } \\
\text { Consistency }\end{array}$ & & & $\begin{array}{l}\text { Internal } \\
\text { Consistency }\end{array}$ & \multirow{2}{*}{\multicolumn{2}{|c|}{$95 \% \mathrm{Cl}$}} & \\
\hline & & Mean & SD & $\begin{array}{l}\text { Cronbach's } \\
\text { Alpha }\end{array}$ & Mean & SD & $\begin{array}{l}\text { Cronbach's } \\
\text { Alpha }\end{array}$ & & & \\
\hline J-KIDSCREEN-52 (self reported) & 52 & & & & & & & & & \\
\hline Physical Well-being & 5 & 31.05 & 10.19 & 0.30 & 52.12 & 12.37 & 0.81 & -24.5 & -17.7 & $<0.00 I^{*}$ \\
\hline Psychological Well-being & 6 & 31.71 & 10.15 & 0.92 & 49.30 & 12.48 & 0.93 & -21.2 & -14.0 & $<0.001 *$ \\
\hline Moods \& Emotions & 7 & 35.22 & 8.75 & 0.82 & 48.40 & 10.82 & 0.87 & -16.2 & -10.2 & $<0.00 I^{*}$ \\
\hline Self-perception & 5 & 40.59 & 6.88 & 0.45 & 45.02 & 9.34 & 0.72 & -6.9 & -2.0 & $<0.001 *$ \\
\hline Autonomy & 5 & 40.61 & 7.40 & 0.65 & 50.01 & 10.23 & 0.83 & -12.1 & -6.8 & $<0.001 *$ \\
\hline Parent Relations \& Home-Life & 6 & 41.12 & 10.86 & 0.86 & 49.44 & 10.11 & 0.90 & -11.5 & -5.1 & $<0.001 *$ \\
\hline Financial Resources & 3 & 43.04 & 11.12 & 0.89 & 47.72 & 11.85 & 0.87 & -8.4 & -1.0 & $0.014 *$ \\
\hline Social Support \& Peers & 6 & 26.33 & 14.95 & 0.91 & 54.62 & 13.20 & 0.92 & -32.8 & -23.8 & $<0.001 *$ \\
\hline School Environment & 6 & 32.67 & 12.68 & 0.49 & 52.14 & 10.58 & 0.86 & -23.8 & -15.1 & $<0.001 *$ \\
\hline Social Acceptance & 3 & 43.33 & 11.94 & 0.65 & 45.93 & 11.02 & 0.71 & -6.3 & 1.1 & 0.171 \\
\hline Predictor variables & & & & & & & & & & \\
\hline RSES & 10 & 25.35 & 8.03 & 0.76 & & & & & & \\
\hline SRAS-R-JA score & 20 & 58.79 & 15.60 & 0.90 & & & & & & \\
\hline
\end{tabular}

Note: $* p<0.05$.

Abbreviations: Cl, confidence interval; SRB, School Refusal Behavior; RSES, Rosenberg Self-esteem Scale; SRAS-R-JA, School Refusal Assessment Scale-Revised for Japanese Attendance at School.

reports, Chau et al reported that SRB (more than 8 days of school absence per year) was associated with poorer physical and psychological HRQOL in middle-school adolescents of the general population, which was measured as lower than the 25 th percentile in the dimensions of physical and psychological of WHOQOL-Bref. ${ }^{16}$ To our knowledge, no study has assessed HRQOL in children with SRB who visited medical services, so our data are relevant to the scientific community. The self (child)reported and proxy-reported HRQOL was significantly

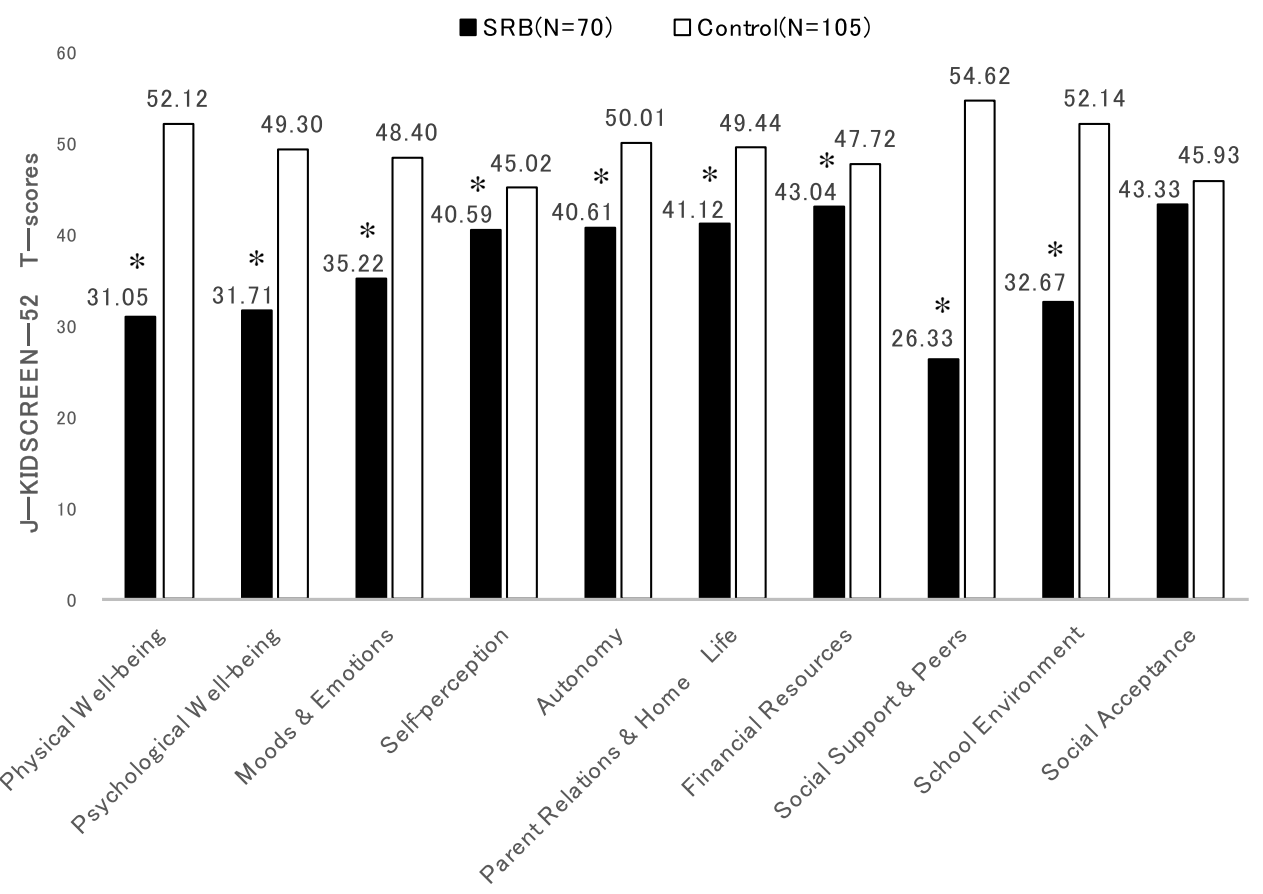

Figure I Health-related quality of life measured by the J-KIDSCREEN-52 in the SRB group compared with the control group. $*_{p}<0.05$. 
Table 4 Difference Between Self-Reported and Proxy-Reported HRQOL and Intraclass Correlation Coefficients Between SelfReported and Proxy-Reported HRQOL

\begin{tabular}{|l|l|l|l|}
\hline $\begin{array}{l}\text { J-KIDSCREEN-52 } \\
\text { Dimensions }\end{array}$ & $\Delta$ & ICC (SRB) & $\begin{array}{l}\text { ICC } \\
\text { (Control) }\end{array}$ \\
\hline Physical Well-being & $4.49 *$ & 0.629 & 0.592 \\
Psychological Well-being & 2.25 & $\mathbf{0 . 3 1 4}$ & 0.492 \\
Moods \& Emotions & $6.17^{*}$ & $\mathbf{0 . 3 4 6}$ & 0.455 \\
Self-perception & $2.43^{*}$ & 0.424 & $\mathbf{0 . 3 6 5}$ \\
Autonomy & -1.42 & $\mathbf{0 . 0 9 4}$ & $\mathbf{0 . 3 8 9}$ \\
Parent Relations \& Home Life & $5.02^{*}$ & $\mathbf{0 . 3 7 6}$ & $\mathbf{0 . 3 7 6}$ \\
Financial Resources & -3.68 & $\mathbf{0 . 3 8 6}$ & 0.474 \\
Social Support \& Peers & -1.09 & 0.541 & 0.456 \\
School Environment & $5.58^{*}$ & 0.603 & 0.529 \\
Social Acceptance & -1.67 & $\mathbf{0 . 2 6 0}$ & 0.521 \\
\hline
\end{tabular}

Notes: A positive $\Delta$ indicates a higher level of HRQOL reported by a child. ${ }^{*} p<0.05$. Values in bold indicate an ICC $<0.40$.

Abbreviations: SRB, School Refusal Behavior; ICC, intraclass correlation coefficient.

different in the dimensions of Moods and Emotions and Parent Relationships and Home Life. The HRQOL was higher in the proxy-reported in the Moods and Emotions dimension, but lower in the Autonomy, Financial Resources, Social Support and Peers, and Social Acceptance dimensions compared to self (child)-reported HRQOL in children with SRB that visited a medical service. Parents and supporters of children should be aware of overestimating of HRQOL when assess their HRQOL. Matza et al reported that self-report measures are preferable whenever feasible for HRQOL measures, ${ }^{33}$ and we therefore considered hearing from the children themselves as important for the assessment of HRQOL. The TDS indicated that $52.2 \%$ of children with SRB had abnormal mental health status, and the J-KIDSCREEN indicated that the SRB group had a lower HRQOL than the control group.

Among all children with SRB that visited medical service, SRB with no psychiatric diagnosis did not associate with the HRQOL. Among the children with SRB who visited medical services, $87.2 \%$ of them had received a psychiatric diagnosis. Prabhuswamy et al reported that, among 33 students with school refusal, 29 subjects $(87.9 \%)$ had a psychiatric diagnosis. ${ }^{10}$ Depression, Adjustment disorder, and somatoform disorder were associated with a lower HRQOL in the dimensions of Physical Well-being, Autonomy, and Social Support and Peers. Conversely, ASD were associated with a higher HRQOL in the Psychological Well-being and Autonomy dimensions. In our study, 9 children (12.9\%) had been diagnosed with ASD in the SRB group. Although the prevalence of ASD in children with SRB or school refusal was different in each medical service and was different between SRB and school refusal, ASD in children with SRB is a recent topic in the treatment of SRB. Suzuki et al reported that $57 \%$ of children that visited the medical service of their university with not-attendance at school were diagnosed with ASD. ${ }^{34}$ Kathrine et al reported that SRB is significantly more common in students with ASD compared with those with typical development, which was assessed by teacher and parent monitoring of students with SRB for 20 days. ${ }^{12}$

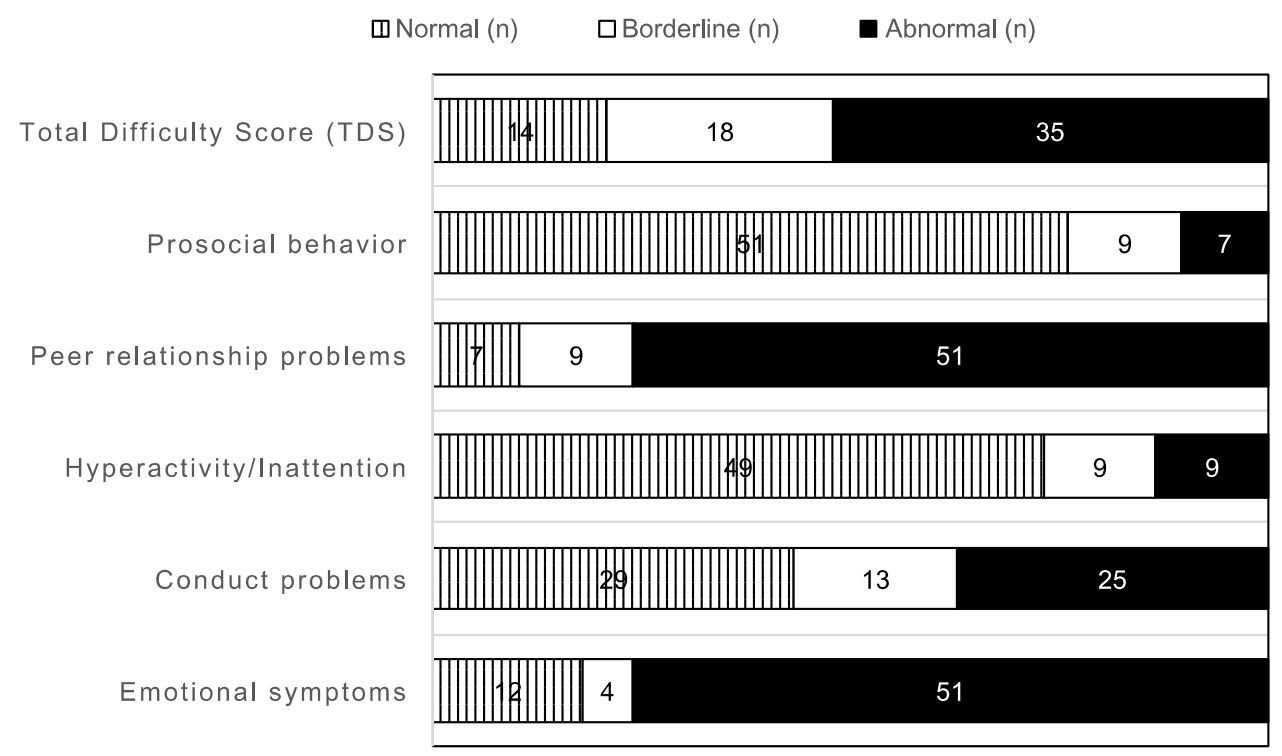

Figure 2 Strengths and difficulties questionnaire in the SRB group.

Abbreviation: TDS, Total Difficulty Score. 
Table 5 Multiple Regression Analysis of the J-KIDSCREEN-52 Dimensions and Factors Associated with HRQOL in SRB Group

\begin{tabular}{|c|c|c|c|c|c|c|c|c|c|c|c|}
\hline & & PH & PW & ME & SP & $\mathbf{A U}$ & PA & FI & PE & SC & SA \\
\hline RSES & & 0.338 & 0.338 & 0.665 & 0.405 & 0.181 & $0.36 I$ & 0.298 & 0.063 & 0.115 & 0.177 \\
\hline SRAS-R-JA score & & -0.027 & 0.055 & 0.001 & -0.113 & 0.036 & 0.189 & 0.075 & -0.378 & $-0.48 I$ & -0.557 \\
\hline ICD-10 & $\begin{array}{l}\text { F3 } \\
\text { F4 } \\
\text { F8 } \\
\text { No diagnosis }\end{array}$ & $\begin{array}{l}-0.229 \\
-0.389 \\
0.003 \\
0.041\end{array}$ & $\begin{array}{l}-0.190 \\
0.036 \\
0.290 \\
0.080\end{array}$ & $\begin{array}{l}-0.187 \\
-0.084 \\
0.144 \\
0.113\end{array}$ & $\begin{array}{l}-0.050 \\
-0.650 \\
0.184 \\
0.067\end{array}$ & $\begin{array}{l}-0.255 \\
0.058 \\
0.262 \\
0.028\end{array}$ & $\begin{array}{l}-0.226 \\
0.051 \\
0.175 \\
0.026\end{array}$ & $\begin{array}{l}0.115 \\
-0.097 \\
0.064 \\
-0.027\end{array}$ & $\begin{array}{l}-0.253 \\
-0.079 \\
0.092 \\
0.160\end{array}$ & $\begin{array}{l}-0.247 \\
0.005 \\
0.101 \\
0.124\end{array}$ & $\begin{array}{l}0.076 \\
0.037 \\
0.128 \\
-0.186\end{array}$ \\
\hline$R^{2}$ & & 0.293 & 0.225 & 0.443 & 0.164 & 0.148 & 0.130 & 0.089 & 0.212 & 0.231 & 0.310 \\
\hline Adjusted $R^{2}$ & & 0.258 & 0.199 & 0.434 & 0.151 & 0.120 & 0.117 & 0.072 & 0.185 & 0.207 & 0.296 \\
\hline
\end{tabular}

Notes: Significant coefficients $(P<0.05)$ are shown in bold. Dichotomous variables: $F 3$ (except for F3 =0,F3 = I), F4 (except for F4 =0,F4 = I), F8 (except for F8 =0, F8 = I), and no diagnosis (any diagnosis $=0$, no diagnosis $=1$ ).

Abbreviations: PH, Physical Well-being; PW, Psychological Well-being; ME, Moods and Emotions; SP, Self-Perception; AU, Autonomy; PA, Parent Relationships and Home Life; Fl, Financial resources; PE, Social Support and Peers; SC, School Environment; SA, Social Acceptance.

The Autonomy dimension of the J-KIDSCREEN-52 is defined as looking at the opportunity given to a child or adolescent to have his/her social and leisure time. High Autonomy scores indicate that they feel free to decide their own day to day activities. The core symptoms of ASD are difficulties in social relationships and communication as core symptoms, as well as anxiety when forming new relationships and avoidance of social situations. ${ }^{35}$ Repetitive patterns of behaviors and compulsion could provoke and maintain SRB, but if their repetitive patterns were accepted by their families or schools, HRQOL in the Autonomy dimension would increase. Munkhaugen et al reported that the difficulty of social motivations in the social responsiveness scale was stronger in patients with ASD who have SRB than in patients with ASD who do not have SRB. ${ }^{36}$ Children with ASD who have SRB feel less conflict about absence from school. This characteristic may explain why children with ASD who have SRB had a higher HRQOL in the Psychological Well-being dimension at the beginning of SRB in the present study. We found that some children with ASD who exhibited SRB had a lower HRQOL after six months than that of their first visit to the medical service in the dimensions of Social Support and Peers and Social Acceptance; we need to follow up the transition of HRQOL (data not shown). Given that children with ASD that have SRB have a gradual decrease in self-esteem, encouraging selfconfidence is important.

Concerning the third hypothesis, higher self-esteem was associated with a higher HRQOL in six dimensions, especially in the Moods and Emotions and Self-Perception dimensions in children with SRB that visited medical services. Our results indicated that self-esteem is a protective factor for HRQOL in children with SRB that visited medical services. In the general population, Otto et al reported that mental health problems were negatively associated with HRQOL, and self-efficacy and social support were positively associated with self-reported HRQOL assessed by the KIDSCREEN-10. ${ }^{13}$ In a cross-sectional study of 723 adolescents (12-18 years of age), Haraldstad et al reported that being involved in bullying, as either the victim or the bully, was associated with a lower HRQOL, as assessed by the KIDSCEEN-52. Previous work has already suggested that increasing self-efficacy might be a way to increase HRQOL in adolescents. ${ }^{14}$ In a study on manualbased cognitive-behavioral treatment for school refusal students, Heyne et al reported that increases in school attendance and decreases in fear about attending school the next day were found to be mediated by self-efficacy posttreatment. ${ }^{15}$

The SRAS-R-JA score was associated with a lower HRQOL in the dimension of Social Support and Peers, School Environment and Social Acceptance in the present study. SRAS-R-JA score is indicative of the persistence of SRB. Thus, lower HRQOL in peer-relationships and school-life may prolong SRB.

Children with SRB diagnosed with a depressive episode, adjustment disorder, or somatoform disorder had a lower HRQOL than that of control group in the dimensions of Physical well-being, Autonomy, and Peer relationships. Thus, children with SRB who are diagnosed with a depressive episode, adjustment disorder, or somatoform disorder should rest well and avoid stress. In contrast, children with SRB diagnosed with ASD in the early stage of SRB 
should be supported according to their neurodevelopmental traits and should adjust their environment rather than resting at home. We found that the HRQOL of children with SRB differed according to the psychiatric disorders of children with SRB in the present study. Thus, making diagnosis of psychiatric disorders is important among children with SRB.

Given that SRB allows them to avoid bad social relationships at school, this can be a way to escape from a crisis. However, this means that they cannot use the educational systems or form good peer-relationships at school. To support children with SRB, psychiatric disorders need to be appropriately diagnosed, and small-step goals concerning the characteristics of each psychiatric diagnosis need to be made. Successful experiences will raise their self-esteem and prevent a decline of HRQOL. Although these strategies are already used in clinical practice, our results support their use.

Given that treatment of children with SRB is considered according to the psychiatric diagnosis, medical services and psychiatrists play a major role in detecting psychiatric disorders, especially in the early stages of SRB. Support that enhances self-esteem could be used to increase HRQOL in young people with SRB. For children with prolonged SRB, the government should provide services that help children with SRB to learn and develop social relationships without attending school. These systems are currently insufficient in Japan.

One strength of this study is that we evaluated children with SRB who visited medical services using standardized HRQOL tool, self-esteem tool, and psychiatric diagnoses. Our results indicate the importance of psychiatric assessment of children with SRB. This study also has some limitations that should be noted. First, there was a selection bias; we selected children who first visited medical services for SRB and recruited children who needed treatment. We did not consider the stage of SRB; participants with various stages of SRB were included, regardless of their first visit to the medical services. Second, the response rate was $56.9 \%$, which means there was a response bias. Third, the sample size was small. Fourth, we could not assess the relationship between HRQOL and the number of days of absence, because we did not measure the days of absence per year. Fifth, the parental health problems difference between control and SRB groups was not considered, and then family context might influence HRQOL of children with SRB. Future work should analyze HRQOL while considering the stage of SRB and the number of days of absence, family context and using a larger sample.

\section{Conclusion}

Our results indicate that children with SRB who visited medical services had a lower HRQOL in many dimensions, and need assistance highly. A psychiatric diagnosis in children with SRB was associated with a lower HRQOL, but a diagnosis of ASD was not associated with a lower HRQOL in patients with SRB. Our results indicate that better self-esteem could enhance HRQOL in children with SRB who visited medical services. Concerning clinical support, we recommend that the presence of psychiatric disorders is assessed in the early stages of SRB, and that support that enhances self-esteem is provided.

\section{Abbreviations}

SRB, school refusal behavior; MEXT, Japanese ministry of education, culture, sports, science and technology; ASD, autism spectrum disorder; HRQOL, health-related quality of life; OSS3, The Oslo 3-item social support scale; PH, Physical Wellbeing; PW, Psychological Well-being; ME, Moods and Emotions; SP, Self-Perception; AU, Autonomy; PA, Parent Relationships and Home Life; FI, Financial Resources; PE, Social Support and Peers; SC, School Environment; SA, Social Acceptance; SD, standard deviation; SRAS-R-JA, school refusal assessment scale-revised for Japanese attendance at school; SRAS-R, the school refusal assessment scale-revised; RSES, Rosenberg self-esteem scale; SDQ, strengths \& difficulties questionnaires; TDS, Total Difficulty Score; ICC, intraclass correlation coefficient.

\section{Data Sharing Statement}

The dataset of this study is available from the corresponding author on reasonable request.

\section{Ethics and Consent Statement}

The present study protocol was approved by the Ethics Committee of Nara Medical University and Nara Prefecture General Medical Center. All children and parents were informed about the purpose of the study before participation. Written informed consent letters were obtained from each participating student and one of the student's parents. This study was conducted in accordance with the Declaration of Helsinki.

\section{Acknowledgments}

We wish to thank the participants for their involvement in the study. The authors would also like to thank the Japanese KIDSCREEN Group for their cooperation in assessment tools and collection of the control group. We 
thank Nia Cason, PhD, from Edanz Group for editing a draft of this manuscript and helping to draft the abstract.

\section{Funding}

This research was supported by the JSPS Grant-in-Aid for Scientific Research (C), 2016-2019 (Hidemi Iwasaka 16K04830).

\section{Disclosure}

All the authors declare that they have no conflicts of interest.

\section{References}

1. Kearney CA, Silverman WK. The evolution and reconciliation of taxonomic strategies for school refusal behavior. Clin Psychol (New York). 1996;3(4):339-354.

2. Kearney CA, Albano AM. The functional profiles of school refusal behavior: diagnostic aspects. Behav Modif. 2004;28(1):147-161. doi:10.1177/0145445503259263

3. Egger HL, Costello JE, Angold A. School refusal and psychiatric disorders: a community study. J Am Acad Child Adolesc Psychiatry. 2003;42(7):797-807. doi:10.1097/01.CHI.0000046865.56865.79

4. Havik T, Bru E, Ertesvåg SK. Assessing reasons for school non-attendance. Scand J Educ Res. 2015;59(3):316-336. doi:10.1080/ 00313831.2014 .904424

5. King NJ, Bernstein GA. School refusal in children and adolescents: a review of the past 10 years. $J$ Am Acad Child Adolesc Psychiatry. 2001;40(2):197-205. doi:10.1097/00004583-200102000-00014

6. Japanese Ministry of Education, Culture, sports, science and technology. [homepage on the Internet]. Available from: http://www.mext. go.jp/en/. Accessed October 23, 2019.

7. McShane G, Walter G, Rey JM. Characteristics of adolescents with school refusal. Aust $N$ Z J Psychiatry. 2001;35(6):822-826. doi:10.1046/j.1440-1614.2001.00955.x

8. Ohmann S, Schuch B, Sackl P, et al. Kognitiv-verhaltenstherapeutische gruppentherapie für jugendliche mit angstbedingter schulverweigerung. Eine beobachtungsstudie. Verhaltenstherapie. 2007;17:175-181. doi:10. $1159 / 000104353$

9. Honjo S, Nishide T, Niwa S, et al. School refusal and depression with school inattendance in children and adolescents. Psychiatry Clin Neurosci. 2001;55:629-634.

10. Prabhuswamy M, Srinath S, Girimaji S, Seshadri S. Outcome of children with school refusal. Indian J Pediatr. 2007;74:375-379. doi:10.1007/s12098-007-0063-5

11. Kurita H. School refusal in pervasive developmental disorders. J Autism Dev Disord. 1991;21(1):1-15. doi:10.1007/BF02206993

12. Kathrine E, Gjevik E, Hugo A, Sponheim E, Diseth T. Research in autism spectrum disorders school refusal behaviour: are children and adolescents with autism spectrum disorder at a higher risk? Res Autism Spectr Disord. 2017;41-42:31-38. doi:10.1016/j.rasd.2017.07.001

13. Otto C, Haller AC, Klasen F, Hölling H, Bullinger M, RavensSieberer U, for BELLA study group. Risk and protective factors of health-related quality of life in children and adolescents: results of the longitudinal BELLA study. PLoS One. 2017;12(12):e0190363. doi:10.1371/journal.pone. 0190363

14. Haraldstad K, Kvarme LG, Christophersen K, Helseth S. Associations between self-efficacy, bullying and health-related quality of life in a school sample of adolescents: a cross-sectional study. BMC Public Health. 2019;19(757). doi:10.1186/s12889-019-7115-4
15. Maric M, Heyne DA, Mackinnon DP. Cognitive mediation of cognitive-behavioural therapy outcomes for anxiety-based school refusal. Behav Cogn Psychother. 2013;41(5):549-564. doi:10.1017/ S1352465812000756

16. Chau K, Kabuth B, Causin-Brice O, et al. Associations between school difficulties and health-related problems and risky behaviours in early adolescence: a cross-sectional study in middle-school adolescents in France. Psychiatry Res. 2016;244:1-9. doi:10.1016/j. psychres.2016.07.008

17. Gonzálvez C, Inglés CJ, Martínez-Palau A, Sanmartín R, Vicent M, García-Fernández JM. Child and adolescent social adaptive functioning scale: factorial invariance, latent mean differences, and its impact on school refusal behavior in spanish children. Front Psychol. 2019;10:1894. doi:10.3389/fpsyg.2019.01894

18. The ICD-10 classification of mental and behavioural disorders: diagnostic criteria for research. World Health Organization; 1993. Available from: https://apps.who.int/iris/handle/10665/37108. Accessed October 23, 2019.

19. Ravens-Sieberer U, Gosch A, Rajmil L, et al. The KIDSCREEN-52 quality of life measure for children and adolescents: psychometric results from a cross-cultural survey in 13 European countries. Value Health. 2008;11(4):645-658. doi:10.1111/j.1524-4733.2007.00291.x

20. The KIDSCREEN Group Europe. The KIDSCREEN Questionnaires -Quality of Life Questionnaires for Children and Adolescents. Lengerich: Pabst Science Publishers; 2006.

21. Nezu S, Iwasaka H, Saeki K, et al. Reliability and validity of the Japanese version of the KIDSCREEN-52 health-related quality of life questionnaire for children/adolescents and parents/proxies. Environ Health Prev Med. 2015;20(1):44-52. doi:10.1007/s12199-014-0427-1

22. Tsuchiya M, Hosotani M, Tojo M. Application of the school refusal assessment scale-revised (SRAS-R) to regular elementary school children and investigation of its validity. Jpn J Behav Ther. 2010;36(2):107-118.

23. Kearney CA. School absenteeism and school refusal behavior in youth: a contemporary review. Clin Psychol Rev. 2008;28 (3):451-471. doi:10.1016/j.cpr.2007.07.012

24. Kearney CA. Identifying the function of school refusal behavior: a revision of the School Refusal Assessment Scale. J Psychopathol Behav Assess. 2002;24:235-245. doi:10.1023/A:1020774932043

25. Kearney CA, Silverman WK. Measuring the function of school refusal behavior: the school refusal assessment scale. J Clin Child Psychol. 1993;22(1):85-96. doi:10.1207/s15374424jccp2201_9

26. Rosenberg M. Society and the Adolescent Self-Image. Prinston University Press; 1965.

27. Yamamoto M, Matsui Y, Yamanari Y. The structure of perceived aspects of self. Jpn J Educ Psychol. 1982;1:64-68.

28. Sakurai S. Investigation of the Japanese version of Rosenberg's Selfesteem Scale. Bull Tsukuba Dev Clin Psychol. 2000;12:65-71.

29. Goodman R, Ford T, Simmons H, Gatward R, Meltzer H. Using the Strengths and Difficulties Questionnaire (SDQ) to screen for child psychiatric disorders in a community sample. Int Rev Psychiatry. 2003;15(1-2):166-172. doi:10.1080/0954026021000046128

30. Goodman R. Psychometric properties of the strengths and difficulties questionnaire. J Am Acad Child Adolesc Psychiatry. 2001;40 (11):1337-1345. doi:10.1097/00004583-200111000-00015

31. Noda W, Ito H, Fujita C, et al. Re-examination of Japanese version of the Strength and Difficulties Questionnaire, Parent Rating Form: generating cut off score and normative data for each grade and sex using the entire cohort data in one suburban city in Japan. Seishin Igaku. 2012;54(4):383-391.

32. Bartko JJ. The intraclass correlation coefficient as a measure of reliability. Psychol Rep. 1966;19(1):3-11. doi:10.2466/pr0.1966.19.1.3

33. Matza LS, Swensen AR, Flood EM, Secnik K, Leidy NK. Assessment of health-related quality of life in children: a review of conceptual, methodological, and regulatory issues. Value Health. 2004;7(1):79-92. doi:10.1111/j.1524-4733.2004.71273.x 
34. Suzuki N, Okayama A, Ohinata J, et al. School refusal as assign of underlying developmental disorders: patient characteristics and prognostic factors. No to Hattatsu. 2017;49:255-259.

35. Rutter M, Taylor E, eds. Child and Adolescent Psychiatry. 4th ed. Oxford: Blackwell Publishing; 2003.
36. Munkhaugen EK, Torske T, Gjevik E, Nærland T, Pripp AH, Diseth TH. Individual characteristics of students with autism spectrum disorders and school refusal behavior. Autism. 2019;23 (2):413-423. doi:10.1177/1362361317748619

\section{Publish your work in this journal}

Neuropsychiatric Disease and Treatment is an international, peerreviewed journal of clinical therapeutics and pharmacology focusing on concise rapid reporting of clinical or pre-clinical studies on a range of neuropsychiatric and neurological disorders. This journal is indexed on PubMed Central, the 'PsycINFO' database and CAS, and is the official journal of The International Neuropsychiatric Association (INA). The manuscript management system is completely online and includes a very quick and fair peer-review system, which is all easy to use. Visit http://www.dovepress.com/testimonials.php to read real quotes from published authors. 